\title{
A utilização da escala de disponibilidade para educação interprofissional em saúde nas publicações nacionais
}

\author{
The use of the availability scale for interprofessional education in health in national \\ publications
}

\author{
Mariana Rotta Bonfim ${ }^{1}$ \\ Orcid: https://orcid.org/0000-0002-8677-1185 \\ Bruna Cunha de Souza ${ }^{2}$ \\ Orcid: https://orcid.org/0000-0002-7464-1500
}

\begin{abstract}
Resumo
Introdução: Visto a crescente discussão acerca do tema da interprofissionalidade, a Readiness for Interprofessional Learning Scale (RIPLS), destaca-se pela eficiência na avaliação e direcionamento de iniciativas de Educação Interprofissional (EIP). Objetivo: Este trabalho objetivou analisar a utilização dessa escala nas pesquisas nacionais. Materiais e Métodos: $\mathrm{O}$ levantamento de artigos foi feito no Pubmed, Periódicos Capes, SCIELO e LILACS utilizando os descritores "Educação Interprofissional", "Educação Superior", "Relações Interprofissionais", "Trabalho em equipe", e "RIPLS". Resultados e Conclusões: Foram identificados 7 artigos que indicam uma tendência de utilização da RIPLS para avaliar iniciativas de EIP na graduação e educação permanente em saúde, sendo escassas as pesquisas sobre a disponibilidade ao longo da formação. Há divergências na versão da RIPLS aplicada e nos métodos de análise, fatos que dificultam a comparação dos estudos. Sugere-se o uso da versão validada dessa ferramenta, a apresentação dos resultados em gráficos e/ou tabelas, e uso de análises estatísticas que garantam a exploração e comparação dos dados.
\end{abstract}

Palavras-chave: educação interprofissional; educação superior; relações interprofissionais

\begin{abstract}
Introduction: In view of the growing discussion on the theme of interprofessionality, the Readiness for Interprofessional Learning Scale (RIPLS), stands out for its efficiency in the evaluation and direction of Interprofessional Education (IPE) initiatives. Objective: This study aimed to analyze the use of this scale in national surveys. Materials and Methods: The survey of articles was done at Pubmed, Capes Journals, SCIELO and LILACS using the descriptors "Interprofessional Education", "Higher Education", "Interprofessional Relations", "Teamwork", and "RIPLS". Results and Conclusions: 7 articles were identified that indicate a tendency to use RIPLS to evaluate EIP initiatives in undergraduate and permanent health education, with little research on availability throughout training. There are divergences in the version of the RIPLS applied and in the methods of analysis, facts that hinder the comparison of the studies. It is suggested to use the validated version of this tool, to present the results in graphs and / or tables, and to use statistical analyzes that guarantee the exploration and comparison of the data.
\end{abstract}

Keywords: interprofessional education; higher education; interprofessional relations

\footnotetext{
${ }^{1}$ Universidade Municipal de São Caetano do Sul -UCS,SP, Brasil. E-mail: mariana.bonfim@prof.uscs.edu.br

${ }^{2}$ Universidade Municipal de São Caetano do Sul -UCS,SP, Brasil. E-mail: brunacsouza98@gmail.com
} 


\section{Introdução}

O Sistema Único de Saúde (SUS) possui entre os seus princípios a integralidade em saúde, entendida como conjunto articulado e contínuo das ações e serviços preventivos e curativos, exigindo para sua efetivação uma prática colaborativa, na qual profissionais de diferentes áreas prestam serviços incluindo todo o contexto social dos pacientes em todos os níveis da rede de serviços. Uma das formas de preparar o profissional para a prática colaborativa é por meio da Educação Interprofissional (EIP), em que estudantes da saúde de diferentes áreas aprendem com os outros, sobre os outros e entre si para efetiva colaboração e melhora dos resultados na saúde ${ }^{1}$.

A aquisição de um ambiente positivo ao efetivo aprendizado interprofissional depende da realização de estudos que permitam conhecer quais propostas interprofissionais se mostram mais efetivas, em que situações foram implantadas e como esses modelos podem impactar nos resultados da atenção à saúde da população, trabalhadores e organizações; logo, dentre as variáveis mais relevantes para serem consideradas na introdução da EIP, estão as atitudes e as expectativas de estudantes diante do trabalho interprofissional $^{2}$.

Dentre as ferramentas utilizadas para obtenção dessas informações destacase a Readiness for Interprofessional Learning Scale (RIPLS), uma escala desenvolvida para avaliar a "prontidão" para a aprendizagem compartilhada em diferentes áreas de atuação. Ela avalia três fatores, sendo o primeiro deles relacionado ao trabalho em equipe, que mostra uma disposição e necessidade de compartilhar conhecimentos e habilidades com outros estudantes, como forma de entender os problemas clínicos no local de trabalho; o segundo fator diz respeito à identidade profissional, que é influenciada pela estrutura e organização acadêmica do graduando; o terceiro e último fator é o papel e responsabilidades que refletem o mapa profissional da área da saúde sendo redesenhado ${ }^{3}$.

Essa escala vem sendo utilizada em diversos países no mundo, o que pode ser comprovado pela quantidade de traduções e validações que vêm sendo realizadas nos últimos anos. De acordo com Oishi et $a l .{ }^{4}$, é importante que a RIPLS tenha sido traduzida para o japonês, porque é esperado que profissionais da saúde liderem a discussão sobre a EIP e a prática colaborativa no país utilizando a escala, possibilitando uma discussão mais aprofundada sobre o tema. Já a validação realizada na Holanda mostrou uma boa confiabilidade para uso na atenção primária, oferecendo novas possibilidades no local de trabalho para o desenvolvimento profissional ${ }^{5}$.

No Brasil, essa escala foi traduzida e validada para o português por Peduzzi et $a l^{2}$, sendo esse processo divido em fase 1 , com a tradução, avaliação pelo comitê de especialistas e pré-testes realizados com estudantes da saúde, e fase 2, na qual foi realizada a validação em português da RIPLS com 347 participantes. O valor 0,60 foi definido como o limite inferior aceitável para o índice de consistência interna alfa de Cronbach, sendo que os resultados alcançados demonstraram consistência interna considerável, obtendo valor do coeficiente de 0,90 e 0,75 para o Fator 1 (Trabalho em equipe e colaboração) e Fator 3 (Atenção à saúde centrada no paciente) respectivamente, sendo que o Fator 2 (Identidade profissional) apresentou valor 0,66; de acordo com a literatura, esses valores são satisfatórios e, portanto, a versão brasileira da RIPLS pode ser considerada um instrumento eficiente de avaliação de iniciativas de EIP, sendo muito útil na formulação de políticas e no 
planejamento de ações em programas interprofissionais ${ }^{2,6}$ (anexo 1).

Assim, considerando a importância da EIP para a prática colaborativa e entendendo a necessidade de utilização de um instrumento de avaliação eficaz para ser utilizado em sua avaliação, este trabalho busca analisar como a RIPLS vem sendo utilizada nas pesquisas brasileiras. Os resultados obtidos com esse levantamento poderão nortear o uso da escala em território nacional e contribuir para futuros estudos na área que venham utilizar esse instrumento de avaliação.

\section{Materiais e Métodos}

\section{Amostra e tipo de estudo}

Este trabalho se caracteriza como uma pesquisa bibliográfica de Revisão Sistemática da Literatura, cujo levantamento de dados se limitou a artigos científicos publicados em revistas indexadas nas bases de dados eletrônicas Pubmed, Periódicos Capes, Scientific Eletronic Library Online (SCIELO) e Literatura Latino-Americana e do Caribe em Ciências da Saúde (LILACS).

\section{Delineamento da pesquisa}

A busca foi realizada nos meses de Janeiro e Fevereiro de 2020, utilizando a combinação dos descritores "Educação Interprofissional", "Educação Superior", "Relações Interprofissionais" e "Trabalho em equipe", sendo selecionadas apenas as pesquisas originais realizadas na população brasileira e que tenham aplicado a Readiness for Interprofessional Learning Scale (RIPLS); para fins de complementação da busca, foi realizado um último rastreamento com o termo "RIPLS".

\section{Critérios de Inclusão e Exclusão}

A seleção dos artigos foi realizada em três etapas: 1) triagem baseada na leitura do título e do resumo, 2) triagem pela leitura na íntegra dos artigos selecionados, 3) triagem pela identificação de estudos referenciados nos artigos selecionados e não encontrados na busca. $\mathrm{O}$ cruzamento dos descritores identificou 381 artigos, dos quais 5 foram selecionados na triagem 1; destes, verificou-se com a leitura na íntegra que apenas 4 artigos atendiam todos os critérios estabelecidos, sendo que o trabalho descartado foi a pesquisa de validação do instrumento para a língua portuguesa.

Com a utilização isolada do termo RIPLS, foram identificados e incluídos neste estudo mais dois artigos em que a escala foi aplicada em população brasileira, resultando, portanto, em seis artigos. Após o levantamento nas referências dos artigos selecionados, foi incluído, mais um trabalho a esta revisão, totalizando sete artigos.

\section{Procedimentos}

Das pesquisas selecionadas foram analisadas as informações sobre a revista, o ano de publicação, o objetivo do trabalho, a amostra do estudo, as características da versão da RIPLS que foi utilizada, os métodos de coleta de dados adicionais, e as estratégias de análise e apresentação dos resultados.

\section{Resultados}

Ao todo foram analisados 7 artigos integralmente (tabela 1), sendo que a análise dos artigos identificados na busca mostra que as pesquisas nacionais que utilizam a RIPLS em seus métodos são prioritariamente publicações de abrangência nacional, visto que apenas um deles foi publicado em revista internacional. De maneira geral, elas começaram a ser publicadas em 2011, sendo as mais recentes de 2018, não tendo periodicidade definida, indicando que esse tema é de interesse recente e crescente.

No que diz respeito aos objetivos dos estudos, verificou-se que a maioria deles é realizado visando identificar os 
impactos da Educação Interprofissional, seja por ações pontuais ou por reorganizações curriculares, na disponibilidade de seus alunos e/ou egressos às experiências e aprendizados interprofissionais ${ }^{7,8,9,10}$. Todavia, também há publicações que avaliaram a diferença dessa disponibilidade em alunos de diferentes cursos e períodos ${ }^{11,12}$, independente de ações de EIP, ou em profissionais formados que atuam no programa Estratégia Saúde da Família (ESF) cuja pós-graduação foi feita em áreas afins ou distintas à saúde pública/da família ${ }^{13}$, indicando que a sua aplicação vai além de avaliar a efetividade de ações de EIP na graduação.

Com relação às amostras estudadas, observa-se uma considerável amplitude no tamanho amostral, variando de 30 a mais de 700 indivíduos, além de extensa diversidade de características da população estudada, englobando diferentes cursos, períodos de formação e status profissional. No que diz respeito ao tamanho amostral, observou-se que as maiores amostras $(n=770, n=545$, $n=135$ e $n=107$ ) foram obtidas nas pesquisas que avaliaram diferentes $\operatorname{cursos}^{7,9,11,12}$, o que amplia a população do estudo; as demais pesquisas realizadas apresentaram uma amostra menor $(n=88$, $\mathrm{n}=34, \mathrm{n}=32)^{8,10,13}$, o que provavelmente está relacionado à avaliação de apenas uma profissão específica. A forma de aplicação da RIPLS, presencialmente ou via formulário por endereço eletrônico, também poderia ser um fator relacionado ao tamanho amostral, visto que se espera que a aplicação presencial aumente a adesão; todavia, a ausência de dados sobre perda amostral nos estudos inviabiliza a identificação da eficácia dos métodos de aplicação.

Tabela 1: Artigos incluídos na pesquisa.

\begin{tabular}{|c|c|c|c|c|c|c|}
\hline Referências & Objetivo & Amostra & RIPLS & $\begin{array}{l}\text { Análises } \\
\text { Adicionais }\end{array}$ & Análise RIPLS & $\begin{array}{c}\text { Apresentação } \\
\text { dos Resultados }\end{array}$ \\
\hline $\begin{array}{l}\text { Aguilar-da- } \\
\text { Silva, R.H.; } \\
\text { Scapin, L.T.; } \\
\text { Batista, N.A. } \\
\text { Revista da } \\
\text { Avaliação da } \\
\text { Educação } \\
\text { Superior, } \\
2011^{6}\end{array}$ & $\begin{array}{c}\text { Avaliar as } \\
\text { percepções dos } \\
\text { estudantes em } \\
\text { relação ao } \\
\text { trabalho em } \\
\text { equipe e a } \\
\text { colaboração nas } \\
\text { atividades do } \\
\text { Programa } \\
\text { Integrador (PI) }\end{array}$ & $\begin{array}{l}\text { N=135 egressos } \\
\text { dos cursos de } \\
\text { Enfermagem, } \\
\text { Fisioterapia, } \\
\text { Farmácia e } \\
\text { Odontologia da } \\
\text { Faculdade de } \\
\text { Ciências } \\
\text { Médicas e de } \\
\text { Saúde de Juiz } \\
\text { de Fora }\end{array}$ & $\begin{array}{l}\text { Versão não } \\
\text { validada com } 09 \\
\text { questões sobre } \\
\text { trabalho em } \\
\text { equipe e } \\
\text { colaboração } \\
\text { com outros } \\
\text { profissionais da } \\
\text { saúde; } \\
\text { Aplicação } \\
\text { inespecífica }\end{array}$ & $\begin{array}{l}\text { Grupo Focal } \\
(\mathrm{n}=10) \\
\text { “o que você } \\
\text { entende por } \\
\text { colaboração } \\
\text { interprofissional } \\
\text { e trabalho em } \\
\text { equipe?” }\end{array}$ & $\begin{array}{l}\text { Frequência } \\
\text { percentual; } \\
\text { soma das } \\
\text { frequências de } \\
\text { discordância e } \\
\text { concordância; } \\
\text { teste de qui- } \\
\text { quadrado para } \\
\text { diferenças entre } \\
\text { grupos (não } \\
\text { aplica) }\end{array}$ & $\begin{array}{c}\text { Apenas no } \\
\text { contexto geral, } \\
\text { sem explorar } \\
\text { diferenças entre } \\
\text { grupos; } \\
\text { Dados } \\
\text { percentuais } \\
\text { descritos no } \\
\text { texto; } \\
\text { Não relaciona } \\
\text { RIPLS e grupo } \\
\text { focal } \\
\end{array}$ \\
\hline $\begin{array}{l}\text { Padula, } \\
\text { M.G.C; } \\
\text { Aguilar-da- } \\
\text { Silva, R.H. } \\
\text { Revista } \\
\text { Odontologia } \\
\text { Unesp, } \\
2014^{12}\end{array}$ & $\begin{array}{c}\text { Analisar } \\
\text { perfil/prática } \\
\text { interprofissional } \\
\text { dos cirurgiões- } \\
\text { dentistas que } \\
\text { atuam na } \\
\text { Estratégia de } \\
\text { Saúde da } \\
\text { Família (ESF) } \\
\end{array}$ & $\begin{array}{c}\mathrm{N}=34 \text { dentistas } \\
\text { que possuem } \\
(\mathrm{n}=26) \text { ou não } \\
(\mathrm{n}=08) \text { pós- } \\
\text { graduação em } \\
\text { saúde pública } \\
\text { ou da família } \\
\text { ESF de } \\
\text { Marília/SP }\end{array}$ & $\begin{array}{l}\text { Adaptação não } \\
\text { validada de } \\
\text { Parsell e Bligh } \\
\text { (1999), com } 35 \\
\text { questões } \\
\text { distribuídas em } \\
4 \text { dimensões*; } \\
\text { Aplicação } \\
\text { inespecífica } \\
\end{array}$ & $\begin{array}{c}\text { Questões } \\
\text { sociodemográfic } \\
\text { as: sexo, idade, } \\
\text { tempo de } \\
\text { atuação no } \\
\text { programa e área } \\
\text { de atuação. }\end{array}$ & $\begin{array}{c}\text { Frequência } \\
\text { percentual; } \\
\text { Kruskal Wallis } \\
\text { com pós-teste } \\
\text { de SNK }\end{array}$ & $\begin{array}{l}\text { Tabelas com } \\
\text { frequência } \\
\text { percentual } \\
\text { categorizada de } \\
\text { acordo com a } \\
\text { pós-graduação, } \\
\text { separadas por } \\
\text { dimensões }\end{array}$ \\
\hline
\end{tabular}




\begin{tabular}{|c|c|c|c|c|c|c|}
\hline $\begin{array}{c}\text { Souto, T.S.; } \\
\text { Batista, } \\
\text { S.H.; } \\
\text { Batista, N.A. } \\
\text { Psicologia: } \\
\text { Ciência e } \\
\text { Profissão, } \\
2014^{7}\end{array}$ & $\begin{array}{c}\text { Avaliar a } \\
\text { proposta de EIP } \\
\text { na UNIFESP } \\
\text { Baixada } \\
\text { Santista na } \\
\text { perspectiva do } \\
\text { estudante de } \\
\text { Psicologia }\end{array}$ & $\begin{array}{l}\mathrm{N}=32 \text { alunos da } \\
\text { psicologia do } 5^{\circ} \\
\text { ano }(\mathrm{n}=22) \text { ou } \\
\text { do } 1^{\circ} \text { ao } 4^{\circ} \text { ano } \\
(\mathrm{n}=10)\end{array}$ & $\begin{array}{c}\text { Versão de } \\
\text { Aguilar-da- } \\
\text { Silva (2011), } \\
\text { porém, com } \\
\text { questões } \\
\text { distintas; } \\
\text { Versão } \\
\text { indefinida; } \\
\text { Aplicação } \\
\text { inespecífica }\end{array}$ & $\begin{array}{c}\text { Grupo Focal: } \\
\text { projeto } \\
\text { pedagógico } \\
\text { interprofissional } \\
\text {, trabalho em } \\
\text { equipe e } \\
\text { integralidade no } \\
\text { cuidado }\end{array}$ & $\begin{array}{c}\text { Pouco } \\
\text { delimitada; } \\
\text { relata } \\
\text { unanimidades / } \\
\text { dados } \\
\text { majoritários de } \\
\text { concordâncias e } \\
\text { discordâncias }\end{array}$ & $\begin{array}{c}\text { Resultados } \\
\text { descritivos no } \\
\text { texto; } \\
\text { Traça relação } \\
\text { entre RIPLS e } \\
\text { grupo focal }\end{array}$ \\
\hline $\begin{array}{c}\text { Rossit, R.; } \\
\text { Batista, } \\
\text { S.H.; } \\
\text { Batista, N.A. } \\
\text { Revista } \\
\text { Internacional } \\
\text { de } \\
\text { Humanidade } \\
\text { s Médicas, } \\
2014^{8}\end{array}$ & $\begin{array}{c}\text { Avaliar a } \\
\text { formação para } \\
\text { integralidade no } \\
\text { cuidado em } \\
\text { carreiras da } \\
\text { saúde na visão } \\
\text { do egresso }\end{array}$ & $\begin{array}{c}\text { N=107 egressos } \\
\text { dos cursos de } \\
\text { Educação } \\
\text { Física, } \\
\text { Fisioterapia, } \\
\text { Nutrição e } \\
\text { Terapia } \\
\text { Ocupacional da } \\
\text { UNIFESP } \\
\text { Baixada } \\
\text { Santista }\end{array}$ & $\begin{array}{c}\text { Versão de } \\
\text { Peduzzi e } \\
\text { Norman (2012) } \\
\text { com } 26 \text { questões } \\
\text { distribuídas em } \\
\text { três } \\
\text { dimensões**; } \\
\text { Escala } \\
\text { hospedada no } \\
\text { Google Docs e } \\
\text { enviada por e- } \\
\text { mail }\end{array}$ & $\begin{array}{l}\text { Questão sobre } \\
\text { satisfação com a } \\
\text { formação } \\
\text { recebida }\end{array}$ & $\begin{array}{l}\text { Frequência } \\
\text { percentual }\end{array}$ & $\begin{array}{c}\text { Gráficos de } \\
\text { frequência } \\
\text { percentual de } 2 \\
\text { questões } \\
\text { específicas; } \\
\text { Tabela com as } \\
09 \text { questões com } \\
\text { maior } \\
\text { concordância } \\
\text { (sem critério) }\end{array}$ \\
\hline $\begin{array}{l}\text { Nuto, S.A.S. } \\
\text { et al. Revista } \\
\text { Brasileira de } \\
\text { Educação } \\
\text { Médica, } \\
2017^{10}\end{array}$ & $\begin{array}{c}\text { Avaliar a } \\
\text { disponibilidade } \\
\text { para } \\
\text { aprendizado } \\
\text { interprofissional } \\
\text { refletindo sobre } \\
\text { a EIP no } \\
\text { currículo } \\
\text { integrado da } \\
\text { Universidade de } \\
\text { Fortaleza. }\end{array}$ & $\begin{array}{c}\mathrm{N}=770 \\
\text { estudantes de } \\
\text { Educação } \\
\text { Física, } \\
\text { Enfermagem, } \\
\text { Farmácia, } \\
\text { Fisioterapia, } \\
\text { Fonoaudiologia, } \\
\text { Odontologia, } \\
\text { Medicina, } \\
\text { Nutrição, } \\
\text { Psicologia e } \\
\text { TO, sendo eles } \\
\text { do primeiro, da } \\
\text { metade e do } \\
\text { último ano. }\end{array}$ & $\begin{array}{c}\text { Versão de } \\
\text { Peduzzi et al. } \\
(2015)^{* * *}, \\
\text { porém relata } 26 \\
\text { questões } \\
\text { distribuídas em } \\
3 \text { dimensões; } \\
\text { Versão } \\
\text { indefinida; } \\
\text { Aplicação } \\
\text { presencial }\end{array}$ & $\begin{array}{c}\text { Questões } \\
\text { sociodemográfic } \\
\text { as: sexo, idade, } \\
\text { curso, tempo de } \\
\text { formação, outra } \\
\text { formação, } \\
\text { participação em } \\
\text { extensão } \\
\text { universitária }\end{array}$ & $\begin{array}{l}\text { Média e Desvio } \\
\text { Padrão dos } \\
\text { escores por fator } \\
\text { e total; } \\
\text { Teste t e Anova } \\
\text { para identificar } \\
\text { diferenças; } \\
\text { Qui-Quadrado } \\
\text { para identificar } \\
\text { as } \\
\text { concordâncias }\end{array}$ & $\begin{array}{c}\text { Tabelas com } \\
\text { valores de média } \\
\text { dos escores dos } \\
\text { fatores e total; } \\
\text { Tabelas } \\
\text { separadas de } \\
\text { acordo com } \\
\text { variáveis de } \\
\text { exposição; } \\
\text { Questões } \\
\text { específicas } \\
\text { apresentadas de } \\
\text { forma descritiva } \\
\text { no texto }\end{array}$ \\
\hline $\begin{array}{l}\text { Tompsen, } \\
\text { N.N. et al. } \\
\text { Revista } \\
\text { Odontologia } \\
\text { UNESP, } \\
2018^{9}\end{array}$ & $\begin{array}{c}\text { Identificar } \\
\text { experiências } \\
\text { curriculares de } \\
\text { EIP e avaliar } \\
\text { disponibilidade } \\
\text { de } \\
\text { estudantes/egres } \\
\text { sos da } \\
\text { graduação em } \\
\text { Odontologia } \\
\text { para o } \\
\text { aprendizado } \\
\text { interprofissional }\end{array}$ & $\begin{array}{c}\mathrm{N}=88, \\
\text { estudantes } \\
(\mathrm{n}=76) \text { ou } \\
\text { egressos }(\mathrm{n}=12 \\
) \text {, concluintes ou } \\
\text { não da } \\
\text { disciplina } \\
\text { integradora em } \\
\text { Universidade } \\
\text { Pública do Sul } \\
\text { do Brasil }\end{array}$ & $\begin{array}{c}\text { Versão de } \\
\text { Peduzzi et al. } \\
(2015) \\
\text { modificada: } 29 \\
\text { questões } \\
\text { originais + } 11 \\
\text { questões dos } \\
\text { autores; } \\
\text { Distribuição em } \\
3 \text { dimensões; } \\
\text { Questionário } \\
\text { enviado por e- } \\
\text { mail }\end{array}$ & $\begin{array}{c}\text { Questões de } \\
\text { perfil } \\
\text { sociodemográfic } \\
\text { o (sexo, idade, } \\
\text { período) e } \\
\text { experiências de } \\
\text { EIP (disciplina } \\
\text { integradora, } \\
\text { estágios } \\
\text { curriculares, } \\
\text { pesquisa, } \\
\text { extensão, } \\
\text { atividades } \\
\text { extras) } \\
\end{array}$ & $\begin{array}{c}\text { Frequência } \\
\text { percentual; } \\
\text { Média e desvio } \\
\text { padrão dos } \\
\text { escores brutos; } \\
\text { Teste t de } \\
\text { Student e } \\
\text { ANOVA. }\end{array}$ & $\begin{array}{c}\text { Tabela } \\
\text { apresentando as } \\
\text { questões, a } \\
\text { distribuição das } \\
\text { frequências e } \\
\text { médias; } \\
\text { Tabelas com } \\
\text { média e DP dos } \\
\text { fatores, } \\
\text { categorizados } \\
\text { por variável } \\
\text { independente }\end{array}$ \\
\hline $\begin{array}{l}\text { Oliveira, } \\
\text { V.F. et al. } \\
\text { Nurse } \\
\text { Education } \\
\text { Today, } \\
2018^{11}\end{array}$ & $\begin{array}{l}\text { Avaliar relação } \\
\text { entre contato de } \\
\text { estudantes de } \\
\text { graduação da } \\
\text { saúde e } \\
\text { resultado de } \\
\text { RIPLS, e } \\
\text { comparar estes }\end{array}$ & $\begin{array}{c}\mathrm{N}=545 \\
\text { estudantes de } \\
\text { Medicina, } \\
\text { Educação } \\
\text { Física, } \\
\text { Enfermagem, } \\
\text { Farmácia, } \\
\text { Fisioterapia, }\end{array}$ & $\begin{array}{c}\text { Versão de } \\
\text { Peduzzi et al. } \\
\text { (2015), porém } \\
\text { relata apenas } 09 \\
\text { questões sobre } \\
\text { Colaboração e } \\
\text { Trabalho em } \\
\text { Equipe; }\end{array}$ & $\begin{array}{c}\text { Questões } \\
\text { Sociodemográfi } \\
\text { cas (idade, sexo, } \\
\text { estado civil, } \\
\text { curso e } \\
\text { período); } \\
\text { Taxa de contato } \\
\text { entre estudantes }\end{array}$ & $\begin{array}{l}\text { Média e desvio } \\
\text { padrão da soma } \\
\text { dos escores; } \\
\text { Anova One } \\
\text { Way e Pós } \\
\text { Teste de } \\
\text { Bonferroni; }\end{array}$ & $\begin{array}{c}\text { Tabelas com } \\
\text { valores de média } \\
\text { e DP dispostos } \\
\text { conforme os } \\
\text { diferentes cursos } \\
\text { e períodos; valor } \\
\text { de p }\end{array}$ \\
\hline
\end{tabular}




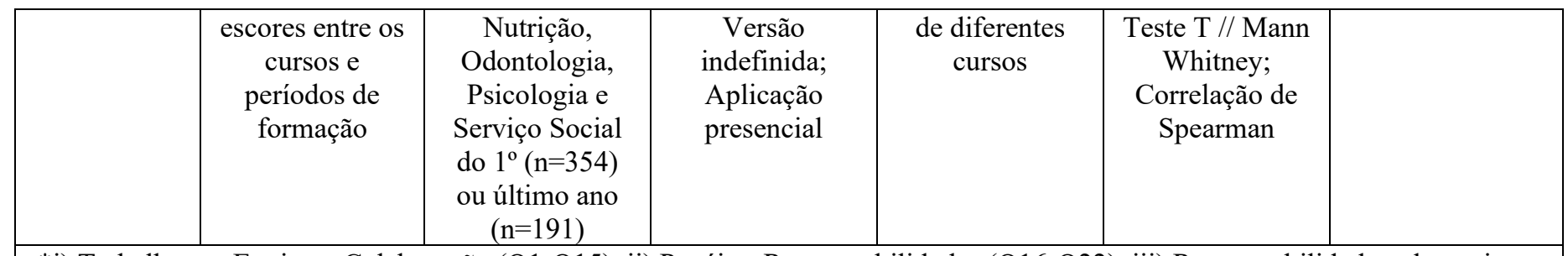

*i) Trabalho em Equipe e Colaboração (Q1-Q15), ii) Papéis e Responsabilidades (Q16-Q22), iii) Responsabilidade pelo paciente (Q23-Q29), iv) Condições para Desenvolvimento do trabalho interprofissional (Q30-Q35); **i) Trabalho em Equipe e Colaboração (Q1-Q10); ii) Identidade Profissional (Q11-Q17); iii) Atenção Centrada no Paciente (Q18-Q26); *** i) Trabalho em Equipe e Colaboração (Q1-Q9, Q12-Q16); ii) Identidade Profissional (Q10,11,17,19,21-24); iii) Atenção Centrada no Paciente (Q25-Q29).

\section{Discussão}

Considerando que o trabalho em equipe faz parte das diretrizes do SUS no Brasil, a RIPLS surge como uma ferramenta importante para futuras formulações de políticas públicas, além de poder auxiliar no planejamento da educação superior em saúde ${ }^{2}$. De fato, Peixoto et al. ${ }^{14}$ afirmam que para a integração da equipe de saúde é fundamental a realização de programas de educação permanente em saúde que tenham como base a interdisciplinariedade, proporcionando uma maior aprendizagem ao profissional e possibilitando uma melhoria nas relações entre paciente, família e equipe, assim como no entendimento do processo saúde-doença, indicando que as ações de EIP não devem ser restritas apenas à graduação. Segundo pesquisa realizada com alunos de uma residência multiprofissional em saúde, além da contribuição para o trabalho em equipe, a EIP proporciona uma satisfação dos profissionais em relação ao respeito mútuo, ao conhecimento da importância do papel do outro e à troca de experiências, gerando um maior aprendizado profissional ${ }^{15}$, mostrando assim a importância de mantê-la na educação permanente em saúde, bem como avaliá-la.

No que diz respeito à quantidade de cursos ou categorias profissionais estudadas, os dados indicam que a RIPLS é uma ferramenta utilizada para avaliar um curso ou área de atuação específica, porém apontam que a maioria dos estudos a utiliza para avaliar diversos cursos ou áreas profissionais simultaneamente, podendo citar Educação Física, Enfermagem, Farmácia, Fisioterapia, Fonoaudiologia, Medicina, Nutrição, Odontologia, Psicologia, Serviço Social e Terapia Ocupacional, viabilizando a ampliação de variáveis de estudo e também do tamanho amostral.

A esse respeito, verificou-se que a Odontologia foi o curso mais explorado no tocante à RIPLS, estando presente em cinco estudos distintos e sendo abordados seus graduandos tanto isoladamente ${ }^{10}$, quanto em comparação com outros cursos ${ }^{7,11,12}$, bem como a atuação de seus profissionais ${ }^{13}$. $\mathrm{Na}$ sequência, observou-se que o curso de Fisioterapia foi avaliado coletivamente em quatro estudos $7,9,11,12$, seguido pela Psicologia em três diferentes estudos, tanto isoladamente ${ }^{8}$ como em comparação com outros $\operatorname{cursos}^{11,12}$, e pelos cursos de Educação Física ${ }^{9,11,12}$, Enfermagem e Farmácia ${ }^{7,11,12}$ que também estiveram presentes em três pesquisas, porém sempre coletivamente. Os cursos de Medicina ${ }^{11,12}$, Nutrição e Terapia Ocupacional ${ }^{9,12}$ foram avaliados coletivamente em dois trabalhos ${ }^{9,11,12}$, sendo os cursos de Fonoaudiologia $^{11}$ e Serviço Social ${ }^{12}$ os menos estudados, estando presentes em apenas um estudo de forma coletiva.

Essa discrepância na frequência de exploração dos cursos pode ter relação com as características de sua oferta. De acordo com o Cadastro Nacional de Cursos e Instituições de Educação Superior (eMEC) $)^{16}$, e considerando apenas o estado de São Paulo, os cursos de Fonoaudiologia e Terapia Ocupacional são oferecidos em cerca de 20 instituições de ensino superior 
cadastradas pelo Ministério da Educação (MEC), números esses bem baixos se comparados com outros cursos como Enfermagem (180 instituições), Educação Física (198 instituições) e Fisioterapia (137 instituições); assim, além de serem menos acessíveis, essa realidade contribui na redução das chances desses cursos estarem vinculados a iniciativas de educação interprofissional em saúde.

Todavia, observa-se que mesmo o curso de Serviço Social sendo oferecido em 113 instituições, ele raramente é avaliado em pesquisas da área da saúde. Tal realidade pode estar relacionada à área acadêmica em que esse curso está inserido, uma vez que suas Diretrizes Curriculares Nacionais são em conjunto com os cursos de Filosofia, História, Geografia, Comunicação Social, Ciências Sociais, Letras, Biblioteconomia, Arquivologia e Museologia, todos considerados da área de humanas. Apesar disso, observa-se que o assistente social está presente na área da saúde, desde a atenção básica até nos serviços de emergência, identificando as mediações e possibilidades dadas pela realidade social, incluindo as demandas institucionais, profissionais e da população que utiliza os serviços de saúde, assim como em questões que interferem no processo saúde e doença ${ }^{17}$.

Esses resultados sugerem que a RIPLS é uma ferramenta importante para estudar as particularidades de uma única área profissional, bem como para explorar as relações existentes entre diferentes cursos e status profissionais, porém apontam a necessidade de mais estudos para aprofundar os conhecimentos referentes às várias profissões que compõem as equipes de saúde. Ainda sobre as amostras estudadas, observou-se que a RIPLS, utilizada em um ou mais cursos, foi também aplicada em diferentes períodos da formação de modo a permitir uma análise comparativa entre os alunos na disponibilidade para a EIP, sendo frequentes as análises do início, meio e/ou final dos cursos, bem como dos egressos.
De maneira geral, as análises obtidas nos primeiros semestres dos cursos têm como objetivo identificar se os alunos já ingressam no ensino superior com perspectivas positivas para a prática colaborativa. A análise dos últimos semestres e/ou de egressos, como no trabalho de Tompsen et al. ${ }^{10}$ com alunos do último ano ou egressos de Odontologia, de Souto, Batista e Batista ${ }^{8}$ com estudantes do $5^{\circ}$ ano de Psicologia, de Aguilar-da-Silva, Scapin e Batista $^{7}$ e Rossit et al. ${ }^{9}$ com egressos de várias graduações, objetivam avaliar as competências de EIP desenvolvidas pelos graduandos ao final do curso. Os trabalhos que comparam os alunos iniciantes e concluintes, como aquele desenvolvido por Oliveira et al. ${ }^{12}$, bem como aqueles que avaliam vários semestres dos cursos com oferta de EIP, como de Nuto et al. $^{11}$, têm por objetivo identificar as diferenças na disponibilidade dos ingressantes e concluintes, sendo constatado que o escore da RIPLS diminui ao longo da formação, indicando que a disponibilidade daqueles que estão concluindo o curso é menor.

De fato, Rapchak et al. ${ }^{18}$ ao avaliarem a eficácia da EIP nos cursos de Farmácia e Enfermagem, evidenciaram que os alunos já iniciam os cursos com atitudes positivas para as práticas colaborativas, ressaltando a importância de que a RIPLS seja aplicada ao longo da graduação, após múltiplas experiências de EIP, para observar se a disponibilidade dos alunos em relação ao assunto se mantém. De maneira semelhante, Montanari ${ }^{19}$ reforça a necessidade de se analisar o comportamento dos estudantes com experiências de aprendizado diferentes nos momentos da formação, contribuindo assim para aumentar o conhecimento sobre a EIP. Essas evidências mostram que há, nas publicações nacionais uma lacuna de conhecimentos, a qual poderia ser sanada pela realização de estudos longitudinais com a RIPLS a fim de aprimorar o acompanhamento dos estudantes até sua formação. 
Vale ressaltar, entretanto, que para o efetivo confronto de dados entre as pesquisas faz-se necessária a utilização de ferramentas padronizadas de avaliação. A esse respeito, o levantamento feito neste estudo mostra que dos sete trabalhos analisados, todos eles utilizam escalas distintas para a avaliação da disponibilidade para EIP. Considerando que o estudo de tradução e validação da RIPLS no Brasil foi publicado em $2015^{2}$, é compreensível que as pesquisas anteriores tenham utilizado versões distintas, como questões específicas de algum fator da escala ${ }^{7,13}$, ou uma versão adaptada da escala original ${ }^{8,9}$; causa estranheza, entretanto, a utilização de outros instrumentos nas publicações mais recentes.

Nesses trabalhos, o que se observa é um relato da utilização da versão validada na apresentação dos métodos, porém no decorrer da explanação verifica-se que as características são distintas. Segundo Peduzzi et $a l^{2}{ }^{2}$, a versão validada para o português possui 27 itens agrupados em três fatores, sendo "Trabalho em Equipe e Colaboração" com 14 questões (1-9 e 1216), "Identidade Profissional" com oito questões (10,11, 17, 19, 21-24), e "Atenção à Saúde Centrada no Paciente" com cinco questões (25-29), tendo sido excluídos os itens 18 e 20 . No trabalho de Nuto et al. ${ }^{11}$, é relatada a utilização da escala validada, porém ele apresenta o instrumento como tendo 26 questões, indicando que provavelmente a versão utilizada foi aquela publicada previamente por Peduzzi e Norman $^{20}$ em um resumo de congresso.

Analogamente, Tompsen et al. ${ }^{10}$ relatam a utilização da escala validada, porém apontam que ela foi modificada, sendo mantidas as 29 questões originais, adicionadas a outras 11 questões elaboradas pelos autores. Oliveira et al. ${ }^{12}$, por sua vez, relatam a utilização apenas do fator Trabalho em Equipe e Colaboração da escala validada, porém apontam que o mesmo é composto por 09 questões; esses dados são conflitantes, uma vez que na escala de Peduzzi et al. ${ }^{2}$ esse fator é composto por 14 itens, indicando que provavelmente a escala utilizada não foi a validada.

De acordo com Alexandre e Coluci $^{21}$, quando são realizadas coletas de dados, é necessária a utilização de instrumentos que garantam indicadores confiáveis, escolhendo assim ferramentas que garantam medidas adequadas e precisas. A validação para o português falado no Brasil feita por Peduzzi et al. $^{2}$, garantiu à escala consistência interna e confiabilidade, demonstrando que a versão é uma ferramenta eficiente para avaliar iniciativas de EIP realizadas em território nacional.

Adicionalmente à heterogeneidade das ferramentas, observa-se uma falta de padronização na forma de análise dos resultados obtidos, contribuindo na dificuldade de confronto dos resultados. De maneira geral, por ser uma escala de Likert, a RIPLS permite a obtenção de dados numéricos sobre a disponibilidade, favorecendo assim a utilização de análises estatísticas robustas que geram resultados mais objetivos. De fato, todos os estudos apresentam os resultados da RIPLS com estatísticas descritivas, sendo a frequência percentual a mais usada; todavia, apenas quatro dos sete artigos utilizam testes de comparação ou correlação para explorar os resultados obtidos ${ }^{10,11,12,13}$. A esse respeito, observa-se que os dados são apresentados e analisados como média dos resultados brutos da escala ou somatória dos fatores, sendo observada maior frequência de diferenças estatísticas nos dados analisados como somatória.

Ao analisarmos a característica dos dados em publicações internacionais, foi observado que Marcussen et al. $^{22}$ apresentaram os resultados obtidos através de tabelas, com o total dos escores, separados pelos fatores da RIPLS, dos grupos estudados, além do $\mathrm{p}$ valor de cada um. Já Mowat et al. ${ }^{23}$ fizeram a utilização de gráficos que comparam os resultados da RIPLS aplicados em três momentos diferentes com profissionais da saúde, 
apresentando a somatória dos fatores da escala, assim como Rapchak et al. ${ }^{18}$ que também aplicaram o questionário em momentos diferentes, no caso, pré e póscurso relacionado à EIP, de forma que expôs os resultados através da média e desvio padrão, novamente separando os mesmos pelos fatores da RIPLS.

No que diz respeito à apresentação dos resultados, observou-se que a maior parte dos estudos opta por utilizar tabelas com valores de frequência, média e desvio padrão dos escores obtidos na escala, tanto de forma isolada ${ }^{10,11,12}$ ou associada à apresentação em gráficos ${ }^{9,13}$; apesar de menos frequente, houve também a apresentação dos resultados apenas de forma descritiva no texto ${ }^{7,8}$. De maneira geral, verificou-se que os trabalhos que utilizaram recursos visuais para apresentar seus resultados facilitaram a visualização e entendimento dos dados, sendo desejável que seja utilizado esse tipo de recuso nos artigos que utilizem a RIPLS.

Além da aplicação dessa escala, todos os estudos fizeram coletas de dados adicionais, sendo frequente a inserção de questões relacionadas ao perfil sociodemográfico dos estudantes, sendo que dos sete artigos analisados quatro obtiveram esses dados, que incluíam informações sobre sexo, idade e o período que estavam na graduação $^{10,11,12,13}$; a inserção de questões relacionadas à experiência de EIP também esteve presente em três trabalhos ${ }^{9,10,11}$. Ademais, dois trabalhos realizaram grupos focais para identificar a percepção da experiência em EIP $^{7,8}$, sendo ainda utilizada uma ferramenta que avalia a taxa de contato entre alunos de diferentes cursos ${ }^{12}$.

\section{Conclusão}

Os resultados deste trabalho indicam que há uma tendência de ampliação na utilização da RIPLS em estudos nacionais que avaliam iniciativas de EIP nos cursos de graduação e na Educação Permanente em Saúde, reforçando a adequação da ferramenta para esse fim; contudo, observase que sua aplicação está sempre associada à utilização de ferramentas adicionais, indicando ser necessária a aquisição de outros dados que complementem sua análise. Observa-se que são escassos os estudos nacionais que utilizam a RIPLS para avaliar a disponibilidade para EIP nos alunos de diferentes cursos da saúde ao longo de sua graduação, sendo esse um importante tema de estudo. Identificou-se, ainda, a presença de divergências em relação à versão da RIPLS aplicada, bem como aos métodos utilizados para sua análise, fatos que dificultam a comparação dos resultados entre os diferentes estudos; nesse sentido, sugere-se que as pesquisas futuras utilizem a versão validada dessa ferramenta, sendo seus resultados apresentados por meio de recursos visuais como gráficos e/ou tabelas, e submetidos a análises estatísticas robustas que garantam a exploração dos dados obtidos, bem como sua comparação com outros estudos.

\section{Referências}

1. Organização Mundial da Saúde. Marco para Ação em Educação Interprofissional e Prática Colaborativa. Genebra; 2010. Disponível em:

http://www.fnepas.org.br/oms_traduzido_2010.pdf. Acesso em: 25 jan. 2020.

2. Peduzzi M, Norman I, Coster $\overline{\mathrm{S}}$, Meireles E. Adaptação transcultural e validação da Readiness for Interprofessional Learning Scale no Brasil. Rev. Esc. Enferm. USP 2015; 49:7-15.

3. Parsell G, Bligh J. The Development of a Questionnaire to Assess the Readiness of Health Care Students for Interprofessional Learning (RIPLS). Med. educ. 1999; 33:95100 . 
4. Oishi A, Haruta J, Yoshimi K, Goto M, Yoshida K, Yoshimoto H. Cross-cultural adaptation of the professional version of the Readiness for Interprofessional Learning Scale (RIPLS) in Japanese. J. interprof. care. 2017; 31:85-90.

5. Pype P, Deveugele M. Dutch Translation and Validation of the Readiness for Interprofessional Learning Scale (RIPLS) in a Primary Healthcare Context. Eur. J. Gen. Pract. 2016; 22:225-31.

6. Freitas ALP, Rodrigues SG. A avaliação da confiabilidade de questionários: uma análise utilizando o coeficiente alfa de Cronbach. XII Simpósio de Engenharia de Produção SIMPEP. Bauru: UNESP, 2005.

7. Aguilar-da-Silva RH, Scapin LT, Batista NA. Avaliação da formação interprofissional no ensino superior em saúde: aspectos da colaboração e do trabalho em equipe. Avaliação (Campinas; Sorocaba) 2011; 16:167-84.

8. Souto TS, Batista SH, Batista NA. A Educação interprofisssional na formação em psicologia: olhares de estudantes. Psicol. ciênc. prof. 2014; 34:32-45.

9. Rossit RAS, Freitas MAO, Batista SSHS, Batista NA. Construção da identidade profissional na educação interprofissional em saúde: percepção de egressos. Interface (Botucatu, Online). 2018; 22:1399-1410.

10. Tompsen NN, Meireles E, Peduzzi M, Toassi, RFC. Educação Interprofissional na graduação em Odontologia: experiências curriculares e disponibilidade de estudantes. Rev. odontol. UNESP (Online) 2018; 45:309-20.

11. Nuto SAS, Lima Júnior FCM, Camara AMCS, Gonçalves CBC. Avaliação da disponibilidade para aprendizagem interprofissional de estudantes de ciências da saúde. Rev. bras. educ. méd. 2017; 41:50-7.

12. Oliveira VF, Bittencourt MF, Pinto IFN, Lucchetti ALG, Ezequiel OS, Lucchetti G. Comparison of the Readiness for Interprofessional Learning and the rate of contact among students from nine different healthcare courses. Nurse educ. today 2018; 63:64-8.

13. Padula MGC, Aguilar-da-Silva RH. Professional profile of dentists who are members of the Family Health Strategy city of Marilia, São Paulo: the challenge of interprofessional work. Rev. odontol. UNESP (Online) 2014; 43:52-60.

14. Peixoto LS, Gonçalves LC, Costa TD, Tavares CMM, Cavalcanti ACD, Cortez EA. Educação permanente, continuada e em serviço: desvendando seus conceitos. Enferm. glob. 2013; 29:324-40.

15. Casanova IA, Batista NA, Moreno LR. A Educação Interprofissional e a prática compartilhada em programas de residência multiprofissional em Saúde. Interface (Botucatu, Online) 2018; 22:1325-37.

16. Cadastro Nacional de Cursos e Instituições de Educação Superior. Brasil: Ministério da Educação [Acesso em: 10 fev. 2020]. Disponível em: http://emec.mec.gov.br/.

17. Neves DCA, Gonçalves C, Favaro TCP. Pós-graduação e Residência Multiprofissional em Saúde HC-UFG: a produção do serviço social. R. Katál. 2017; 20:225- 33.

18. Rapchak ME, Nolfi DA, Turk MT, Marra L, O’Neil CK. Implementing an interprofessional information literacy course: impact on student abilities and attitudes. $J$. med. libr. assoc. 2018; 106:464-70.

19. Montanari PM. Formação para o trabalho no ensino das graduações em saúde. Saúde Soc. 2018; 27:980-86.

20. Peduzzi M, Norman IJ. Validação de instrumento de medida do aprendizado interprofissional para trabalho em equipe. Encontro Internacional de Pesquisa em Enfermagem. São Paulo: EE-USP, 2012.

21. Alexandre NMC, Coluci MZO. Validade de conteúdo nos processos de construção e adaptação de instrumentos de medidas. Cien. Saude Colet. 2011; 16:3061-68. 
22. Marcussen M, Nørgaard B, Borgnakke K, Arnfred S. Interprofessional clinical training in mental health improves students' readiness for interprofessional collaboration: a nonrandomized intervention study. BMC med. educ. 2019; 27:1-10.

23. Mowat S, Hein C, Walsh T, MacDonald L, Grymonpre R, Sisler J. Changing health professionals' attitudes and practice behaviors through interprofessional continuing education in oral-systemic health. J. dent. educ. 2018; 81:1421-29.

\section{Como citar este artigo:}

Bonfim MR, Souza BC. A utilização da escala de disponibilidade para educação interprofissional em saúde nas publicações nacionais. Rev. Aten. Saúde. 2021; 19(67): 369380 . 
Anexo 1. Itens e fatores que compõem a escala Readiness for Interprofessional Learning Scale (RIPLS)

\begin{tabular}{|c|c|c|c|c|c|}
\hline & 1 & 2 & 3 & 4 & 5 \\
\hline \multicolumn{6}{|l|}{ TRABALHO EM EQUIPE E COLABORAÇÃO } \\
\hline \multicolumn{6}{|l|}{$\begin{array}{l}\text { 1. A aprendizagem junto com outros estudantes ajudará a me tornar um participante mais } \\
\text { efetivo de uma equipe em saúde. }\end{array}$} \\
\hline \multicolumn{6}{|l|}{$\begin{array}{l}\text { 2. Em última análise os pacientes seriam beneficiados se estudantes da área da saúde } \\
\text { trabalhassem juntos para resolver os problemas dos pacientes. }\end{array}$} \\
\hline \multicolumn{6}{|l|}{$\begin{array}{l}\text { 3. Aprendizagem compartilhada com outros estudantes da área da saúde aumentará minha } \\
\text { capacidade de compreender problemas clínicos. }\end{array}$} \\
\hline \multicolumn{6}{|l|}{$\begin{array}{l}\text { 4. A aprendizagem junto com outros estudantes da área da saúde durante a graduação } \\
\text { melhoraria os relacionamentos após a graduação. }\end{array}$} \\
\hline \multicolumn{6}{|l|}{$\begin{array}{l}\text { 5. Habilidades de comunicação deveriam ser aprendidas junto com outros estudantes da área } \\
\text { da saúde }\end{array}$} \\
\hline \multicolumn{6}{|l|}{$\begin{array}{l}\text { 6. A aprendizagem compartilhada me ajudará a pensar positivamente sobre outros } \\
\text { profissionais }\end{array}$} \\
\hline \multicolumn{6}{|l|}{$\begin{array}{l}\text { 7. Para que a aprendizagem em pequenos grupos funcione, os estudantes precisam confiar e } \\
\text { respeitar uns aos outros. }\end{array}$} \\
\hline \multicolumn{6}{|l|}{$\begin{array}{l}\text { 8. Habilidades de trabalho em equipe são essenciais na aprendizagem de todos os estudantes } \\
\text { da área da saúde }\end{array}$} \\
\hline \multicolumn{6}{|l|}{ 9. A aprendizagem compartilhada me ajudará a compreender minhas próprias limitações. } \\
\hline \multicolumn{6}{|l|}{$\begin{array}{l}\text { 12. Habilidades para solução de problemas clínicos só devem ser aprendidas com estudantes } \\
\text { do meu próprio curso. }\end{array}$} \\
\hline \multicolumn{6}{|l|}{$\begin{array}{l}\text { 13. A aprendizagem compartilhada com estudantes de outras profissões da saúde ajudará a me } \\
\text { comunicar melhor com os pacientes e outros profissionais. }\end{array}$} \\
\hline \multicolumn{6}{|l|}{$\begin{array}{l}\text { 14. Gostaria de ter a oportunidade de trabalhar em projetos, em pequenos grupos, com } \\
\text { estudantes de outras profissões. }\end{array}$} \\
\hline \multicolumn{6}{|l|}{$\begin{array}{l}\text { 15. A aprendizagem compartilhada ajudará a esclarecer a natureza dos problemas dos } \\
\text { pacientes. }\end{array}$} \\
\hline $\begin{array}{l}\text { 16.A aprendizagem compartilhada durante a graduação me ajudará a tornar-me um } \\
\text { profissional que trabalha melhor em equipe. }\end{array}$ & & & & & \\
\hline
\end{tabular}

\section{IDENTIDADE PROFISSIONAL (IdP)}

10.Não quero desperdiçar meu tempo aprendendo junto com estudantes de outras profissões da saúde.

11. Não é necessário que estudantes de graduação da área da saúde aprendam juntos.

17. A função dos demais profissionais da saúde é principalmente apoio aos médicos.

19. Preciso adquirir muito mais conhecimentos e habilidades que estudantes de outras profissões da saúde.

21. Eu me sentiria desconfortável se outro estudante da área da saúde soubesse mais sobre um tópico do que eu.

22. Serei capaz de usar frequentemente o meu próprio julgamento no meu papel profissional (autonomia profissional).

23. Chegar a um diagnóstico será a principal função do meu papel profissional (objetivo clínico).

24. Minha principal responsabilidade como profissional será tratar meu paciente (objetivo clínico).

\section{ATENÇÃO À SAÚDE CENTRADA NO PACIENTE (AS)}

25. Gosto de entender o problema na perspectiva do paciente (situação do paciente).

26. Estabelecer uma relação de confiança com meus pacientes é importante para mim (situação do paciente).

27. Procuro transmitir compaixão aos meus pacientes (situação do paciente).

28. Pensar no paciente como uma pessoa é importante para indicar o tratamento correto (situação do paciente).

29. Na minha profissão são necessárias habilidades de interação e cooperação com os pacientes (situação do paciente). 